\title{
On Raman spectra of water, its structure and dependence on temperature
}

\author{
S.M. Baschenko, L.S. Marchenko \\ Institute of Physics, NAS of Ukraine \\ 46, prospect Nauky, 03028 Kyiv, Ukraine \\ E-mail: baschenk@iop.kiev.ua
}

\begin{abstract}
Raman spectra of water within the temperature range 20 to $3{ }^{\circ} \mathrm{C}$ were investigated. Best of all, the complex shape of the obtained spectra was approximated by four (or five) Gaussian-shaped peaks with their positions 3070, 3230, 3440, 3600 (and $3650) \mathrm{cm}^{-1}$. The most intensive constituents were 3230 and $3440 \mathrm{~cm}^{-1}$ lines, their total contribution to the Raman spectrum of water is higher than $90 \%$. These lines are the most sensitive to the temperature, too. When the temperature decreases from 20 down to $3{ }^{\circ} \mathrm{C}$, the positions of these peaks were shifted from 3232 to $3224 \mathrm{~cm}^{-1}$ and from 3441 to $3434 \mathrm{~cm}^{-1}$, respectively; and the intensity of $3230 \mathrm{~cm}^{-1}$ line was increased approximately by $14 \%$ in its magnitude.
\end{abstract}

Keywords: Raman spectra, water structure, temperature, pressure.

Manuscript received 07.06.10; accepted for publication 02.12.10; published online 28.02.11.

\section{Introduction}

Investigations of water, its structure and properties are actual and important due to the role that water plays at the Earth and in human life. Such investigations have been carried out for many years. But up to date, there is no total clearance in understanding and explanation of the water structure, its dependence on environment, the main parameters of which are temperature and pressure $[1,2]$.

"Water clusters" - groups of water molecules held together by hydrogen bonds - have been the subject of a number of intensive experimental and theoretical investigations [3] because of their importance for understanding the water properties.

The most informative and non-disturbing method to investigate various media is the Raman scattering of light in them $[4,5]$. Since its discovery in the 1920s, the Raman spectroscopy has become an important tool for understanding the nature of hydrogen bonds. Raman spectroscopy is called a "fingerprint" technique - every chemical element gives a unique Raman signal, or spectrum. Hundreds of books have been published concerning the fingerprints of various chemical compounds. Raman spectroscopy provides the possibility to conclude that the water molecules retain their molecular character in every phase of $\mathrm{H}_{2} \mathrm{O}$ and that the four-coordinated tetrahedral configuration of water molecules is preserved in each phase.

\section{Experimental setup}

For these investigations, the authors have created a typical multi-pass optical cuvette, in which provided were the changes within the ranges: from $+30{ }^{\circ} \mathrm{C}$ down to $-10^{\circ} \mathrm{C}$ for temperature and from 1 to $100 \mathrm{~atm}$ for pressure. The scheme of this cuvette is presented in Fig. 1. As seen from this figure, the exciting laser beam is directed into the cuvette through the window 1 (or 2 in dependency on experimental geometry) under an angle $\alpha$ (slightly less than $90^{\circ}$ ) to the cuvette axis in such a manner that maximum possible reflections of the beam from the mirrors 3 and 4 occurred. These mirrors 3 and 4 form the so-called optical wedge with the angle $\beta$ near the top. These angles $\alpha$ and $\beta$ may be preliminary adjusted in some intervals and were chosen in the way that the exciting beam passes a maximum possible length in a medium under study up to its full absorption. The beam scattered under the angle of $90^{\circ}$ to the cuvette axis (when dealing with Raman, Rayleigh, Mie 
scattering, etc.) exits from the cuvette through the window 5 and is collected by a lens to the receiving system. To increase the useful signal, the additional mirror 6 was used. Our receiving system consists of a double monochromator MSA-130 and the connected to it photomultiplier FEU-106 that operates in "photon counting" mode.

For the best heat conduction, the cuvette was fabricated of aluminum. But its inner surface was coated with stainless foil thus providing the contacts of liquid under study only with stainless steel, teflon and glass (or silica). Such cuvette construction makes it possible to investigate not only water or neutral liquids, but also the aggressive ones.

Outer sides of the cuvette were equipped with several thermoelectric coolers that provides changes in temperature inside the cuvette within the range +30 to $10{ }^{\circ} \mathrm{C}$. Due to negligible water compressibility, the pressure change inside the cuvette from 0 to $100 \mathrm{~atm}$ is available by using the stainless diaphragm.

\section{Results}

Raman spectra of distilled water were excited by laser irradiation with wavelengths 488 and $532 \mathrm{~nm}$. The scattered Raman signal was collected at the angle close to $90^{\circ}$ relatively to the exciting laser beam, then directed to a double monochromator and detected by the photomultiplier ФЭУ-106 operating in the "photon counting" mode. It should be mentioned that shorter wavelengths provides a higher Raman signal but simultaneously higher background. Therefore, there exists optimum in the signal-to-noise ratio for wavelengths of exciting light. We did not find this optimum, but for the wavelengths 488 and $532 \mathrm{~nm}$ we did not observe any difference.

It is well known that Raman spectra of water have a complex structure [4]. Typical obtained Raman spectra of water are presented in Figs 2 and 3. It is clearly seen that with great precision the complex spectrum shape may be approximated by four or five Gaussian-shaped constituents: two main and two or three minor ones. Positions of maxima for these five constituents are close to $3070,3230,3440,3600$ and $3650 \mathrm{~cm}^{-1}$. The most intense constituents are 3230 and $3440 \mathrm{~cm}^{-1}$ lines that contribute more than $90 \%$ to the Raman spectrum of water. The obtained experimental results for 20 and $3{ }^{\circ} \mathrm{C}$ are presented in Figs 4 and 5, respectively. The lines peaking at 3230 and $3440 \mathrm{~cm}^{-1}$ proved to be the most sensitive to change with temperature. So, when decreasing the temperature from 20 down to $3{ }^{\circ} \mathrm{C}$, the following changes in these two lines were detected:

- positions of their maxima move from 3232 to $3224 \mathrm{~cm}^{-1}$ and from 3441 to $3434 \mathrm{~cm}^{-1}$, respectively;

- relative magnitude of the line $3230 \mathrm{~cm}^{-1}$ was increased by $14 \%$.
All the rest constituents give less than $10 \%$ contribution to the total Raman spectrum intensity, and determination of their number, magnitudes and maximum positions in the conditions of our experiments were very unreliable.

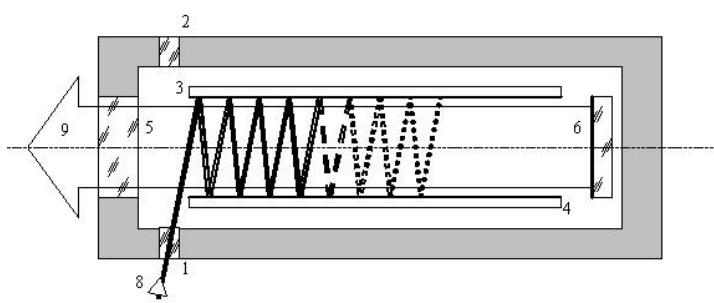

Fig. 1. Scheme of the cuvette for liquid in our Raman scattering investigations: $1,2-$ entrance windows, 3,4mirrors of the "optical wedge", 5 - exit window, 6 - additional mirror, 8 - exciting light, 9 - scattered light.

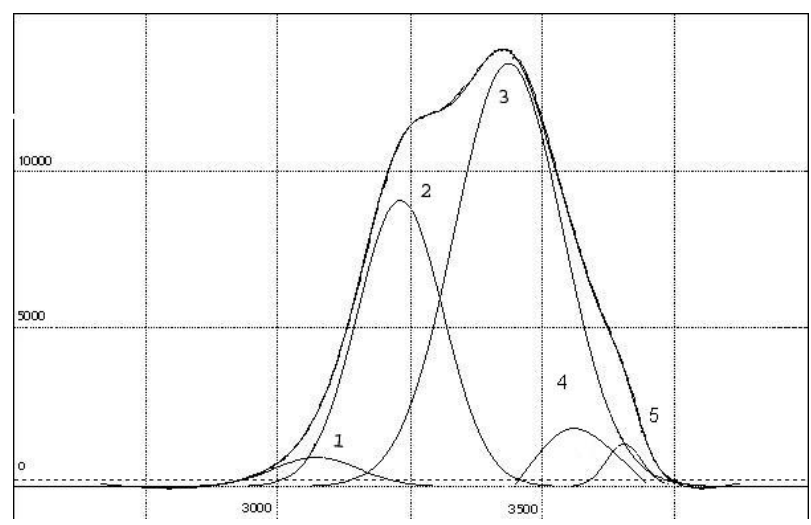

Fig. 2. Typical complex Raman spectrum of water, approximated by five constituents with positions of peaks near: 1 - 3074, 2 - 3233, 3 - 3437, $4-3596$ and $5-3666 \mathrm{~cm}^{-1}$. Main constituents 3230 and $3440 \mathrm{~cm}^{-1}$ form more than $90 \%$ of the total spectrum area, always present and were detected reliably.

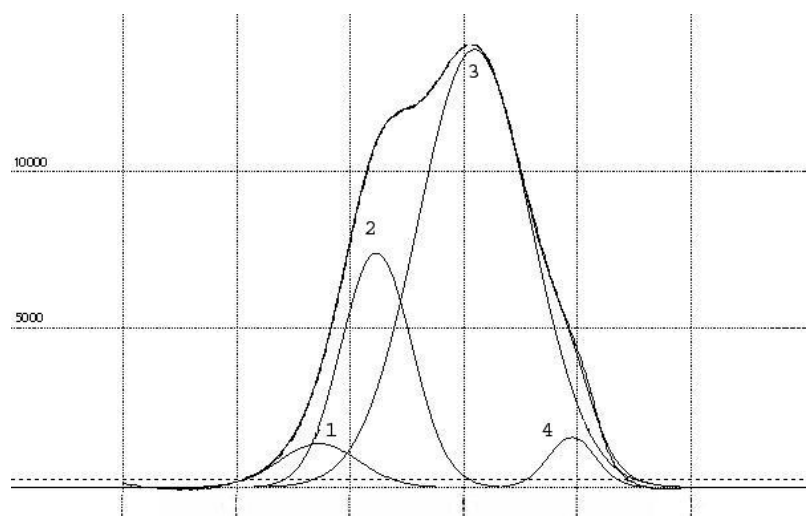

Fig. 3. Typical complex-shaped Raman spectrum of water decomposed by four constituents: $1-631,3 \mathrm{~nm} ; 2-642,3 \mathrm{~nm}$; $3-651 \mathrm{~nm} ; 4-659,5 \mathrm{~nm}$ under pumping by $533 \mathrm{~nm}$. It is obvious that two or three minor ones do not play significant role in spectrum approximation. 


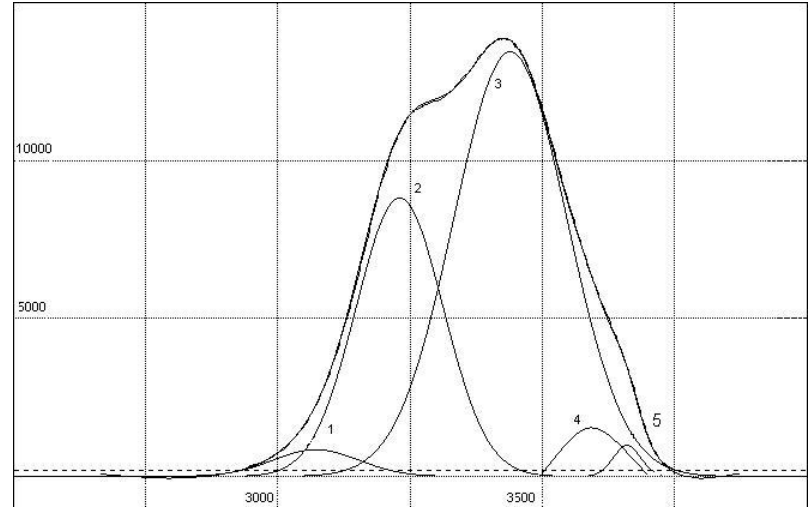

Fig. 4. Obtained Raman spectrum of water at the temperature $20{ }^{\circ} \mathrm{C}$. Positions of constituents: $1-3073,5 ; 2-3231,9 ; 3-$ $3441 ; 4-3611,6$ and $5-3661,5 \mathrm{~cm}^{-1}$.

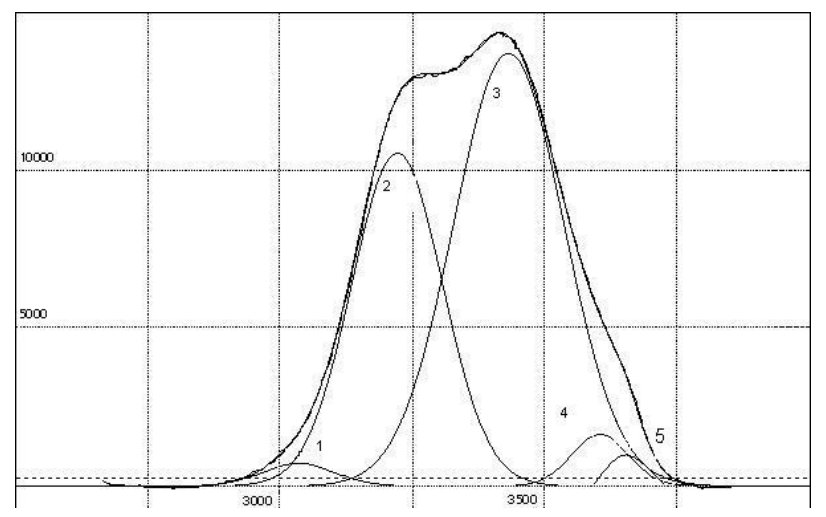

Fig. 5. Obtained Raman spectrum of water at the temperature $3{ }^{\circ} \mathrm{C}$. Positions of constituents: $1-3036,7 ; 2-3223,1 ; 3-$ $3434 ; 4-3606,7$ and $5-3657,2 \mathrm{~cm}^{-1}$. It is clearly seen that the magnitude of $3230 \mathrm{~cm}^{-1}$ line has been increased and positions of 3230 and $3440 \mathrm{~cm}^{-1}$ lines have been shifted by $7-8 \mathrm{~cm}^{-1}$ into the shortwave range.

\section{Conclusion}

The structure of Raman spectra of distillated water as well as their dependency on temperature within the range 20 to $3{ }^{\circ} \mathrm{C}$ and pressure up to $100 \mathrm{~atm}$ were investigated. The revealed shift up to $7-8 \mathrm{~cm}^{-1}$ for positions of two main constituents in short-wave direction can be explained as "freezing" the vibrations. And the changes in their amplitude relation can be explained as water structure redistribution. The pressure increase up to $100 \mathrm{~atm}$ causes no changes in the Raman spectra structure.

\section{References}

1. S. Freeman, Properties of Water, in: Biological Science. SE, Pearson Prentice Hall Inc., 2006.

2. Ch.L. Braun, S.N. Smirnov, Why is water blue? // J. Chem. Educ. 70 (8), p. 612 (1993).

3. D.W. Ball, Rayleigh and Raman Scattering // Spectroscopy 9(6), p. 20-21 (1994).

4. D.A. Long, The Raman Effect. John Wiley \& Sons, New York, 2002. 